\title{
Potential and field singularity at a surface point charge
}

Alexander Silbergleit ${ }^{\mathrm{a})}$

Gravity Probe B, W. W. Hansen Experimental Physics Laboratory, Stanford University, Stanford, California 94305-4085

Ilya Mandel ${ }^{\text {b) }}$

Physics Department, Mail Code 103-33, California Institute of Technology, Pasadena, California 91125

Ilya Nemenman ${ }^{\text {c) }}$

Kavli Institute for Theoretical Physics, University of California, Santa Barbara, California 93106

(Received 14 May 2003; accepted 13 June 2003)

The behavior of the magnetic potential near a point charge (fluxon) located at a curved regular boundary surface is shown to be essentially different from that of a volume point charge. In addition to the usual inverse distance singularity, two singular terms are generally present. The first of them, a logarithmic one, is axially symmetric with respect to the boundary normal at the charge location, and proportional to the sum of the two principal curvatures of the boundary surface at this point, that is, to the local mean curvature. The second term is asymmetric and proportional to the difference of the two principal curvatures in question; it is also bounded at the charge location. Both terms vanish, apparently, if the charge is at a planar point of the boundary, and only in this case. The field in the charge vicinity behaves accordingly, featuring generally two singular terms proportional to the inverse distance, in addition to the main inverse distance squared singularity. This result is significant, in particular, for studying the interaction of magnetic vortices in type II superconductors. (C) 2003 American Institute of Physics.

[DOI: $10.1063 / 1.1605497$ ]

Magnetic vortex lines are formed in type II superconductors. ${ }^{1}$ When crossing the superconductor boundary, they create strongly localized surface sources of magnetic field (fluxons), which may play an important role in various physical situations. For instance, two space tests of Einstein's General Relativity, Gravity Probe B, ${ }^{2,3}$ and STEP (Space Test of the Equivalence Principle), ${ }^{4}$ are based on low temperature technology with type II superconductors, and their setup is significantly affected by fluxons.

The size of a surface magnetic spot is about the microscopic London length, ${ }^{1}$ i.e., it is typically much smaller than characteristic macroscopic sizes involved. Thus the point charge approximation appears naturally and proves to be sufficient for many applications. Within this approximation, the magnetic potential, $\psi=\psi(\mathbf{R})$, satisfies the Neumann boundary value problem

$$
\begin{gathered}
\Delta \psi=0, \quad \mathbf{R} \in D \\
\left.\frac{\partial \psi}{\partial n}\right|_{S}=\sum_{j=1}^{N} \nu_{j} \Phi_{0} \delta_{S}\left(\mathbf{R}-\mathbf{R}_{j}\right), \quad \mathbf{R}, \mathbf{R}_{j} \in S
\end{gathered}
$$

Here the domain $D$ is the empty space, surface $S$ is the superconductor boundary, $\Phi_{0}=h / 2 e$ is the

\footnotetext{
a)Electronic mail: gleit@relgyro.stanford.edu

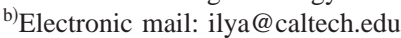

${ }^{c)}$ Electronic mail: nemenman@kitp.ucsb.edu
} 
magnetic flux quantum in SI units, and the magnetic field is $\mathbf{B}=-\nabla \psi$. Moreover, $\delta_{S}\left(\mathbf{R}-\mathbf{R}_{j}\right)$ denotes the surface delta-function at the position, $\mathbf{R}_{j}$, of a vortex, and $\nu_{j}$ is either plus or minus one, depending on whether the field line enters the domain $D\left(\nu_{j}=+1\right)$, or exits it $\left(\nu_{j}=-1\right)$. We assume that the boundary $S$ is smooth enough (at least $C^{3}$ ) near every charge. Outside the charge vicinities it may have any singularities compatible with the finite local energy condition, meaning $(\nabla \psi)^{2}$ is locally integrable.

If $D$ is bounded, then each vortex line starts and ends at the boundary, the number of charges is even, and the total charge vanishes, $\Sigma_{j=1}^{N} \nu_{j} \Phi_{0}=0$, which condition is the solvability criterion of the problem, Eqs. (1) and (2). If the domain $D$ is infinite, some field lines may end at infinity, and this condition may not hold; in any case, we do not use it in the following analysis, which is entirely local.

An immediate question regarding the above boundary value problem is how does its solution behave near a surface charge? For a curved boundary, an answer based on the similarity with the volume point charge turns out incorrect. This is seen from the simplest example, a spherical domain. A closed-form exact solution to Eqs. (1) and (2) in the exterior of a sphere was obtained in Ref. 5. It shows that a new logarithmic singular term, inversely proportional to the radius of the sphere, is added to the main inverse distance singularity in the expansion of the potential near the charge. So, what happens with the singularity for a generally curved smooth surface?

Our search for the answer to this natural and, in fact, classical question covered books and papers in both mathematical physics and in the field of vortices in superconductors, as well as communications with colleagues in both fields. We also talked with high energy theorists expecting to find perhaps some relevant results in view of the discussions of the magnetic monopole. However, no ready answer was found, which might not be so surprising. Indeed, the Neumann boundary value problem with surface charges is not relevant to the design of electrostatic systems. On the other hand, its magnetostatic implementation became available only with the widespread technical use of superconductors in the recent years. Last but not least, the answer proves to be not that simple.

In this paper we fill the gap by deriving a complete singular part of the expansion of the solution to Eqs. (1) and (2) near a charge at an arbitrary curved smooth boundary. As compared to the case of a sphere, one more singular term, proportional to the difference of the two principal curvatures, appears in the general case.

We are interested in the behavior of the potential near a single surface charge at some $\mathbf{R}_{j}$. For brevity, we thus drop the charge index in the following calculation. We put the origin of a Cartesian coordinate system at $\mathbf{R}_{j}$, so that $\mathbf{r} \equiv \mathbf{R}-\mathbf{R}_{j}$. We point the $z$ axis along the outward normal to the surface $S$ (that is, into the superconducting bulk), choosing the $x$ and $y$ axes in the tangent plane, so that the unit vectors $\{\hat{x}, \hat{y}, \hat{z}\}$ form a right orthogonal triplet. Along with Cartesian $\{x, y, z\}$, we will use the corresponding spherical, $\{r, \theta, \phi\}$, and cylindrical, $\{\rho, \phi, z\}$, coordinate systems (see Fig. 1).

The shape of the smooth boundary surface in the vicinity of the charge can be described by the equation $z=F(x, y)$. The Taylor expansion of the function $F(x, y)$ around $x=y=0$ apparently has no terms linear in $x$ or $y$, since $z$ is oriented along the normal. Moreover, by an appropriate rotation of the coordinate axes $\hat{x}, \hat{y}$ in the tangent plane, we can ensure that the second crossderivative of $F$ vanishes at the origin, hence the expansion acquires the form

$$
z=F(x, y)=\frac{k^{(x)}}{2} x^{2}+\frac{k^{(y)}}{2} y^{2}+O\left(\rho^{3}\right) \equiv f(x, y)+O\left(\rho^{3}\right),
$$

where

$$
k^{(x)}=\left.\frac{\partial^{2} F}{\partial x^{2}}\right|_{x=y=0}, \quad k^{(y)}=\left.\frac{\partial^{2} F}{\partial y^{2}}\right|_{x=y=0}
$$

are the two principal curvatures of the boundary surface at the charge location. 


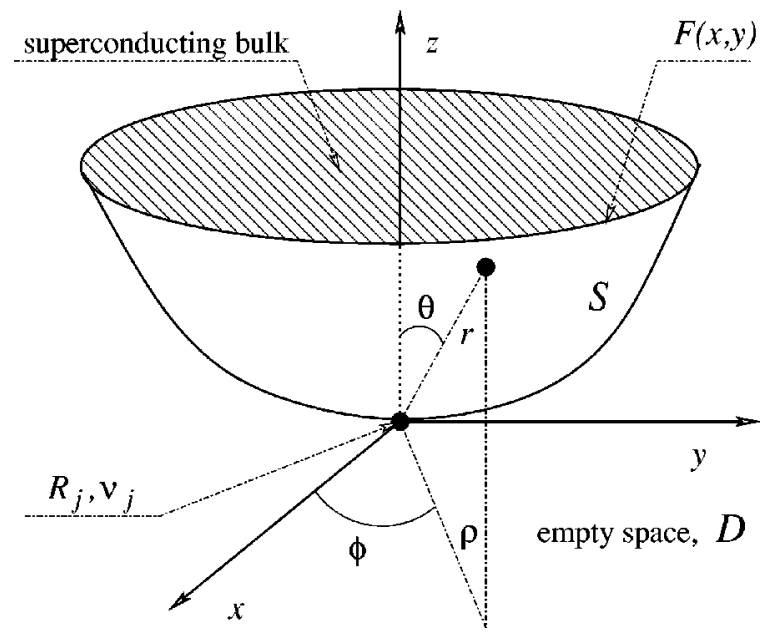

FIG. 1. Definition of coordinate systems near a charge.

Since, near $\mathbf{r}=0$,

$$
\begin{gathered}
\delta_{S}(\mathbf{r})=\delta(x) \delta(y) / J \\
\partial / \partial n=\hat{n} \cdot \nabla=(1 / J)\left(\partial / \partial z-F_{x} \partial / \partial x-F_{y} \partial / \partial y\right), \\
J \equiv \sqrt{1+F_{x}^{2}+F_{y}^{2}}
\end{gathered}
$$

the boundary condition, Eq. (2), in the vicinity of the charge can be written in terms of variables $x, y, z$ as ${ }^{6}$

$$
\left.\frac{\partial \psi}{\partial z}\right|_{z=F(x, y)}=\nu \Phi_{0} \delta(x) \delta(y)+\left.\left(F_{x} \frac{\partial \psi}{\partial x}+F_{y} \frac{\partial \psi}{\partial y}\right)\right|_{z=F(x, y)} .
$$

The partial derivatives of the function $F(x, y)$ near the origin are given, to the order we are interested in, by

$$
F_{x}=k^{(x)} x+O\left(\rho^{2}\right), \quad F_{y}=k^{(y)} y+O\left(\rho^{2}\right) .
$$

Once again, we only care about the vicinity of the charge where $z=F(x, y)$ is small, so we can use perturbation of the boundary to move the boundary condition, Eq. (5), to the plane $z=0$. This is done by means of the following Taylor expansion of an arbitrary function $w=w(x, y, z)$ :

$$
\left.w\right|_{z=F(x, y)}=\left.w\right|_{z=0}+\left.F \frac{\partial w}{\partial z}\right|_{z=0}+\left.\frac{F^{2}}{2} \frac{\partial^{2} w}{\partial z^{2}}\right|_{z=0}+\cdots .
$$

Applying this to the derivatives of $\psi$ in Eq. (5) we write it, to the proper order, in the form:

$$
\left.\frac{\partial \psi}{\partial z}\right|_{z=0}=\nu \Phi_{0} \delta(x) \delta(y)+\left.\left(F_{x} \frac{\partial \psi}{\partial x}+F_{y} \frac{\partial \psi}{\partial y}-F \frac{\partial^{2} \psi}{\partial z^{2}}\right)\right|_{z=0}+\cdots .
$$

The final step of this derivation is to expand $\psi$ in a series of successively smaller (that is, less singular at the origin) functions $\psi^{(i)}$,

$$
\psi=\psi^{(0)}+\psi^{(1)}+\psi^{(2)}+\cdots .
$$


Introducing this expansion in the boundary condition Eq. (7) and then matching the terms of the same order, we end up with the following sequence of boundary conditions for $\psi^{(i)}$, $i=0,1,2, \ldots$, at $z=0$ :

$$
\begin{gathered}
\left.\frac{\partial \psi^{(0)}}{\partial z}\right|_{z=0}=\nu \Phi_{0} \delta(x) \delta(y), \\
\left.\frac{\partial \psi^{(1)}}{\partial z}\right|_{z=0}=\left.\left[k^{(x)} x \frac{\partial \psi^{(0)}}{\partial x}+k^{(y)} y \frac{\partial \psi^{(0)}}{\partial y}-f(x, y) \frac{\partial^{2} \psi^{(0)}}{\partial z^{2}}\right]\right|_{z=0},
\end{gathered}
$$

and so on. Here we have dropped higher order terms on the right-hand sides by replacing $F$ and $F_{x}, F_{y}$ with their main term expressions from Eqs. (3) and (6), respectively. Of course, all functions $\psi^{(i)}$ are subject to the Laplace equation, Eq. (1).

Thus, locally we have successfully replaced the boundary value problem of Eqs. (1) and (2) in the domain $D$ by a sequence of problems for functions $\psi^{(i)}, i=0,1,2, \ldots$, harmonic in the half-space $z<0$ and satisfying the above boundary conditions, Eqs. (9), (10), etc. We now need to solve these problems for the half-space one by one, until the normal derivative of the solution becomes finite at the boundary.

The zero-order solution $\psi^{(0)}$ obeying the boundary condition of Eq. (9) is, of course,

$$
\psi^{(0)}=\frac{\nu \Phi_{0}}{2 \pi} \frac{1}{r} .
$$

It allows one to immediately calculate the right-hand side of Eq (10). Indeed,

$$
\left.f \frac{\partial^{2} \psi^{(0)}}{\partial z^{2}}\right|_{z=0}=-\left.\frac{\nu \Phi_{0} f}{2 \pi}\left(\frac{1}{r^{3}}-\frac{3 z^{2}}{r^{5}}\right)\right|_{z=0}=-\frac{\nu \Phi_{0}}{2 \pi} \frac{k^{(x)} x^{2}+k^{(y)} y^{2}}{2 \rho^{3}},
$$

where the second term in the middle expression turns to zero at $z=0$, contributing no $\delta$-like singularities, due to the presence of the factor $f=O\left(\rho^{2}\right)$. Also taking into account that $\partial r^{-1} / \partial x$ $=-x / r^{3}, \partial r^{-1} / \partial y=-y / r^{3}$, we find the boundary condition for $\psi^{(1)}$ in its final explicit form:

$$
\left.\frac{\partial \psi^{(1)}}{\partial z}\right|_{z=0}=-\frac{\nu \Phi_{0}}{2 \pi} \frac{k^{(x)} x^{2}+k^{(y)} y^{2}}{2 \rho^{3}}=-\frac{\nu \Phi_{0}}{8 \pi}\left[\frac{k^{(x)}+k^{(y)}}{\rho}+\frac{k^{(x)}-k^{(y)}}{\rho} \cos 2 \phi\right] .
$$

The two terms on the utmost right here have essentially different singularities at the origin. For this reason, we treat them separately by splitting the problem in two in the following way:

$$
\begin{gathered}
\psi^{(1)}=\psi_{s}^{(1)}+\psi_{r}^{(1)}, \\
\left.\frac{\partial \psi_{s}^{(1)}}{\partial z}\right|_{z=0}=-\frac{\nu \Phi_{0}}{8 \pi} \frac{k^{(x)}+k^{(y)}}{\rho}, \\
\left.\frac{\partial \psi_{r}^{(1)}}{\partial z}\right|_{z=0}=-\frac{\nu \Phi_{0}}{8 \pi} \frac{k^{(x)}-k^{(y)}}{\rho} \cos 2 \phi .
\end{gathered}
$$

The Neumann problem for $\psi_{s}^{(1)}$ in the half-space does not have solutions bounded at infinity, as one would expect in our investigation (we are actually looking for terms growing away from the charge, because a weaker singularity next to the inverse distance is most probably some logarithm tending to infinity at both the charge and the infinite distance from it). For this reason, no solution can be found by means of standard techniques. However, a harmonic and regular in the half-space $z<0$ function 


$$
\begin{gathered}
\psi_{s}^{(1)}=K_{+} \ln [(r-z) / d]=K_{+}[\ln (r / d)+\ln (1-\cos \theta)], \\
K_{ \pm} \equiv \nu \Phi_{0}\left[k^{(x)} \pm k^{(y)}\right] / 8 \pi,
\end{gathered}
$$

where $d>0$ is an arbitrary constant of the dimension of length, provides the needed solution. Indeed, it satisfies the boundary condition, Eq. (14), in view of

$$
\partial \ln (r-z) / \partial z=(r-z)^{-1}(z / r-1)=-1 / r \rightarrow-1 / \rho, \quad z \rightarrow-0 .
$$

The solution given by Eq. (16) is unique in the class of functions with the logarithmic growth at infinity, namely, those with the asymptotics

$$
\psi_{s}^{(1)}=K_{+} \ln (r / d)+K_{+} \ln (1-\cos \theta)+o(1), \frac{\partial \psi_{s}^{(1)}}{\partial r}=K_{+} / r+O\left(1 / r^{2}\right), \quad r \rightarrow \infty .
$$

Contrary to the previous one, the Neumann problem for $\psi_{r}^{(1)}$,

$$
\Delta \psi_{r}^{(1)}=0, \quad z<0,\left.\frac{\partial \psi_{r}^{(1)}}{\partial z}\right|_{z=0}=-\frac{K_{-}}{\rho} \cos 2 \phi,
$$

has a unique, up to a constant, solution bounded at infinity [namely, a solution that obeys somewhat unusual conditions $\left.\psi_{r}^{(1)}=O(1), \partial \psi_{r}^{(1)} / \partial r=o\left(1 / r^{2}\right), r \rightarrow \infty\right]$. The solution is obtained by the standard separation of variables in cylindrical coordinates using the Hankel transform, and it reads:

$$
\begin{aligned}
\psi_{r}^{(1)} & =-K_{-} \cos 2 \phi \int_{0}^{\infty} J_{2}(\lambda \rho) \exp (-\lambda|z|) \frac{d \lambda}{\lambda} \\
& =-\frac{K_{-} \cos 2 \phi}{2}\left(\frac{\rho}{r-z}\right)^{2}=-\frac{K_{-}}{2} \frac{x^{2}-y^{2}}{(r-z)^{2}} .
\end{aligned}
$$

The value of the integral is found in Ref. 7, 4.14.(5), and the constant $K_{-}$is defined in Eq. (17). Interestingly, this solution in spherical coordinates does not depend on the radius, being a function of the angles only [singular on the positive semiaxis $z>0$, same as $\psi_{s}^{(1)}$ in Eq. (16)]:

$$
\psi_{r}^{(1)}=-\frac{K_{-}}{2} \frac{\sin ^{2} \theta \cos 2 \phi}{(1-\cos \theta)^{2}}, \quad \frac{\partial \psi_{r}^{(1)}}{\partial r}=0
$$

It is now straightforward to see that the Neumann boundary data for all higher order corrections to the potential, starting with $\psi^{(2)}$, are finite at the origin (and dropping fast enough at infinity); accordingly, the solutions of the corresponding problems bounded at infinity are unique up to an additive constant. It also means that all the terms in the expansion, Eq. (8), of the potential, whose normal derivative are singular at the location of a surface charge, are given by the solutions already found. Hence, combining the expressions from Eqs. (11), (16), and (19), we find the desired formula for the magnetostatic potential near a surface charge $(r \rightarrow 0)$ :

$$
\begin{aligned}
\psi & =\psi^{(0)}+\psi_{s}^{(1)}+\psi_{r}^{(1)}+\cdots \\
& =\frac{\nu \Phi_{0}}{2 \pi}\left[\frac{1}{r}+\frac{k^{(x)}+k^{(y)}}{4} \ln \frac{r-z}{d}-\frac{k^{(x)}-k^{(y)}}{8} \frac{x^{2}-y^{2}}{(r-z)^{2}}\right]+(\text { nonsingular terms }) .
\end{aligned}
$$

It is easy to rewrite this in our general notations from Eqs. (1) and (2) by replacing $|\mathbf{r}|$ with $\left|\mathbf{R}-\mathbf{R}_{j}\right|, x$ with $X-X_{j}$, etc. Instead, we give the expression of the singular part of the magnetic field near the charge. It can be written in the form: 


$$
\begin{aligned}
\mathbf{B}= & -\nabla \psi=\frac{\nu \Phi_{0}}{2 \pi}\left[\frac{\hat{r}}{r^{2}}-\frac{k^{(x)}+k^{(y)}}{4 r}\left(\hat{r}+\frac{\sin \theta}{1-\cos \theta} \hat{\theta}\right)-\frac{k^{(x)}-k^{(y)}}{4 r} \frac{\sin \theta}{(1-\cos \theta)^{2}}(\cos 2 \phi \hat{\theta}\right. \\
& +\sin 2 \phi \hat{\phi})]+ \text { (nonsingular terms) }
\end{aligned}
$$

Here are a few concluding remarks regarding the obtained result.

First, notice that the leading order contribution to the potential, Eq. (11), is twice that of the point charge located in a volume away from its boundaries. This is clearly explained by the fact that the field lines and the flux from the surface charge emanate only into the half-space, versus the full space for the volume charge.

The two singular corrections to the usual inverse distance singularity of the potential, Eq. (20), are very different. The first one is logarithmic, axially symmetric about the direction of the normal to the boundary at the charge location, and proportional to the sum of two principal surface curvatures there, i.e., to the mean boundary curvature. Thus, it vanishes if the charge sits at a symmetric saddle point of the boundary. The second additional singularity is asymmetric, proportional to the difference of the principal curvatures, and vanishes thus when the latter are equal, i.e., when the charge is at a spherical point of the boundary. This second term is bounded at the charge location [giving unbounded field components, see Eq. (21)], but is not uniquely defined there, with the limiting values depending on the direction along which the limit is taken. Note that both corrections vanish simultaneously if and only if the charge is at the planar point of the boundary.

In a particular case when the domain $D$ is the exterior of a sphere of the radius $a$, one has $k^{(x)}=k^{(y)}=1 / a$. If there is just one surface charge, $N=j=1$ and $\nu=1$ (so that the incoming vortex line ends at infinity), Eq. (20) becomes

$$
\psi=\frac{\Phi_{0}}{2 \pi}\left[\frac{1}{\left|\mathbf{R}-\mathbf{R}_{1}\right|}+\frac{1}{2 a} \ln \frac{\left|\mathbf{R}-\mathbf{R}_{1}\right|-\hat{n} \cdot\left(\mathbf{R}-\mathbf{R}_{1}\right)}{d}\right]+(\text { nonconstant nonsingular terms }),
$$

in complete agreement with the exact solution obtained in Ref. 5 with $d=2 a$.

Finally, the obtained singular expansion of the potential can be used in the derivation of the force acting on a charge in a fashion similar to the one developed in the case of volume point charges, ${ }^{8}$ i.e., by means of the geometrical regularization of energy and, henceforth, the force, as the energy gradient in the charge location. However, in a striking contrast with the volume case, the force here is found to depend on the gradient of the curvature at the charge location. Namely, due to the first additional singular term in the potential, Eq. (20), there appears a tangential force on the charge which tries to move it toward the point of the stationary mean curvature of the boundary, and which diverges in the regularization limit. If confirmed, this divergence would mean that either the approximation of the point surface charges does not completely describe real microscopic, but finite size fluxons, or, strangely enough, that the fluxons cannot reside at arbitrary points of a curved boundary, or perhaps even something else.

A detailed study of fluxon interactions will be carried out in a separate publication. However, it is clear that it will necessarily use the results of this paper, in view of the relation

$$
\psi(\mathbf{r}, \mu)=\int_{S} d S(\boldsymbol{\xi}) \mu(\boldsymbol{\xi}) \psi(\mathbf{r}-\boldsymbol{\xi})
$$

where $\psi(\mathbf{r}, \mu)$ is the potential created by the surface charge density $\mu(\boldsymbol{\xi}), \boldsymbol{\xi} \in S$, and $\psi(\mathbf{r})$ is the potential from Eq. (20). For small, yet finite size fluxons the divergent asymptotics derived above will have an explicit short scale cutoff defined by the spatial extent of the density (presumably, the London length). However, the detailed analysis will require a deeper insight in the real structure of magnetic vortex lines near a boundary. Without such an analysis one cannot, in fact, speculate about the strength and importance of these surface interactions; we will thus limit ourselves to just a few short comments. 
First, one compares, naturally, the surface force coming from the logarithmic term in the field potential to the strength of the random pinning force that defines the fluxon's position. ${ }^{9}$ The latter depends on the flux tube length and the former does not. So, allowing for a physical regularization of the mathematically divergent surface effects, one will in any case come up with some characteristic length, $L$, below which the surface force will dominate. The description of the vortex line dynamics that does not account for surface effects at distances from the surface smaller than $L$ is necessarily incomplete.

Second, forces between two vortices in a superconducting bulk are exponentially small if the vortex line separation is larger than the London length (precisely the regime we are discussing). These forces can be neglected. Thus, the surface effects we have found will be the leading interaction terms. Such effects are significant and translate into an experimentally relevant magnetic "friction" between superconducting bodies. ${ }^{10}$

We thank Leonid Bakaleinikov, David Gross, Lev Kapitanski, Akakii Melikidze, Andrei Ruckenstein, Andrey Shytov, and Robert Wagoner for discussions and valuable references. A.S. and partly I.M. were supported by NASA Grant No. NAS 8-39225 to Gravity Probe B. I.N. was supported by NSF Grant No. PHY99-07949 to Kavli Institute for Theoretical Physics.

\footnotetext{
${ }^{1}$ M. Tinkham, Introduction to Superconductivity (McGraw-Hill, New York, 1996).

${ }^{2}$ C. W. F. Everitt, in Near Zero, edited by J. D. Fairbank, B. S. Deaver, Jr., C. W. F. Everitt, and P. F. Michelson (Freeman, New York, 1988), pp. 585-639.

${ }^{3}$ D. Bardas et al., in Proceedings of the Eighth Marcel Grossman Meeting on General Relativity, edited by T. Piran (World Scientific, Singapore, 1999), part B, pp. 1135-1138.

${ }^{4}$ J. Mester et al., Class. Quantum Grav. 18, 2475 (2001).

${ }^{5}$ I. M. Nemenman and A. S. Silbergleit, J. Appl. Phys. 86, 614-624 (1999).

${ }^{6}$ Although the Neumann boundary condition should not, in general, allow for a solution for a single vortex, we avoid this complication by extending below the domain $D$ away from the fluxon.

${ }^{7}$ H. Bateman and A. Erdélyi, Tables of Integral Transforms (McGraw-Hill, New York, 1954), Vol. 1.

${ }^{8}$ A. Silbergleit, I. Nemenman, and I. Mandel, Tech. Phys. 48, 146 (2003).

${ }^{9}$ G. Blatter et al., Rev. Mod. Phys. 66, 1125 (1994).

${ }^{10}$ G. Sapilewski, Ph.D. thesis, Department of Aeronautics and Astronautics, Stanford University, 2000.
} 